\title{
PLASMA COMPOSITION IN A SIGMOIDAL ANEMONE ACTIVE REGION
}

\author{
D. Baker ${ }^{1}$, D. H. Brooks ${ }^{2}$, P. Démoulin ${ }^{3}$, L. van Driel-Gesztelyi ${ }^{1,3,4}$, L. M. Green ${ }^{1}$, K. Steed ${ }^{5}$, And J. Carlyle ${ }^{1}$ \\ ${ }^{1}$ University College London, Mullard Space Science Laboratory, Holmbury St Mary, Dorking, Surrey, RH5 6NT, UK \\ ${ }^{2}$ College of Science, George Mason University, 4400 University Drive, Fairfax, VA 22030, USA \\ ${ }^{3}$ Observatoire de Paris, LESIA, CNRS, F-92195 Meudon, France \\ ${ }^{4}$ Konkoly Observatory, Hungarian Academy of Sciences, Budapest, Hungary \\ ${ }^{5}$ Centre for Mathematical Plasma Astrophysics, KU Leuven, Celestijnenlaan 200B, B-3001 Leuven, Belgium \\ Received 2013 August 1; accepted 2013 September 30; published 2013 November 6
}

\begin{abstract}
Using spectra obtained by the EUV Imaging Spectrometer (EIS) instrument onboard Hinode, we present a detailed spatially resolved abundance map of an active region (AR)-coronal hole $(\mathrm{CH})$ complex that covers an area of $359^{\prime \prime} \times 485^{\prime \prime}$. The abundance map provides first ionization potential (FIP) bias levels in various coronal structures within the large EIS field of view. Overall, FIP bias in the small, relatively young AR is 2-3. This modest FIP bias is a consequence of the age of the AR, its weak heating, and its partial reconnection with the surrounding $\mathrm{CH}$. Plasma with a coronal composition is concentrated at AR loop footpoints, close to where fractionation is believed to take place in the chromosphere. In the AR, we found a moderate positive correlation of FIP bias with nonthermal velocity and magnetic flux density, both of which are also strongest at the AR loop footpoints. Pathways of slightly enhanced FIP bias are traced along some of the loops connecting opposite polarities within the AR. We interpret the traces of enhanced FIP bias along these loops to be the beginning of fractionated plasma mixing in the loops. Low FIP bias in a sigmoidal channel above the AR's main polarity inversion line, where ongoing flux cancellation is taking place, provides new evidence of a bald patch magnetic topology of a sigmoid/flux rope configuration.
\end{abstract}

Key words: solar wind - Sun: abundances - Sun: filaments, prominences

Online-only material: animation, color figures

\section{INTRODUCTION}

Elemental abundances of the Sun and their spatial and temporal variations are crucial to our understanding of the physical processes inherent to space weather as they can be used to probe the source regions of the solar wind (SW) and to trace the SW throughout interplanetary space. Furthermore, composition can provide clues to the magnetic topology of the SW source regions as plasma on open magnetic fields can be distinguished from plasma confined in closed loops (Woo et al. 2004; Laming 2004; Wang et al. 2009). As demonstrated by van Driel-Gesztelyi et al. (2012), the magnetic topology of active regions (ARs) bordering coronal holes $(\mathrm{CHs})$ has significant implications for coronal outflows and the SW.

In a review of spectroscopic measurements of the abundances in the solar atmosphere, Meyer (1985a, 1985b) determined that the elemental abundance variation observed in the solar corona and SW compared with the photosphere strongly depends on the first ionization potential (FIP) of the element. No other systematic trend could be identified with any other parameters, e.g., mass and charge (Meyer 1991). Elements can be divided into low-FIP and high-FIP groups with the step at approximately $10 \mathrm{eV}$. Those elements with low FIPs (e.g., Mg, $\mathrm{Si}, \mathrm{Fe}$ ) are enhanced in the corona and the $\mathrm{SW}$ by a factor of 3-4 over photospheric abundances, whereas high-FIP elements (e.g., C, O, Ne, S) maintain the elemental distribution of the photosphere. This phenomenon is known as the FIP effect. In order to characterize the abundance variations of the solar upper atmosphere, typically the corona, the FIP bias ( FIP $\left._{\text {Bias }}\right)$ is used to define the ratio of the elemental abundance in the solar atmosphere $\left(A_{\mathrm{SA}}\right)$ to the elemental abundance in the photosphere $\left(A_{\mathrm{Ph}}\right)$ such that $\mathrm{FIP}_{\mathrm{Bias}}=A_{\mathrm{SA}} / A_{\mathrm{Ph}}$.

Though the composition of the photosphere is well known and seemingly invariant, the composition of the corona and the SW varies substantially from structure to structure and with time. In general, the elemental abundances of the corona are closely related to its morphology (Feldman \& Widing 2002). The FIP bias of $\mathrm{CHs}$ has been determined to be $\sim 1$, i.e., photospheric in composition (Feldman \& Widing 1993; Doschek et al. 1998; Feldman et al. 1998; Brooks \& Warren 2011), whereas quiet Sun (QS) plasma was found to vary from $\sim 1.5$ to 3.5 , depending on the time in the solar cycle, the height above the limb, and the methods used to measure FIP bias (Feldman \& Widing 1993; Doschek et al. 1998; Warren 1999; Landi et al. 2006).

The active Sun provides considerable FIP bias variation. AR plasma confined to loops at the time of flux emergence showed photospheric composition (Sheeley 1995, 1996; Widing 1997; Widing \& Feldman 2001); however, within a few hours, the FIP bias progressively increased to reach coronal abundances after 2 days (Widing \& Feldman 2001). The FIP bias levels of more established ARs ( $\sim$ few days) were 4.8-5.9 (Widing \& Feldman 1995), and old ARs observed for at least 7 days had FIP bias values ranging from 8 to 16 (Feldman 1992; Widing \& Feldman 1995, 2001; Young \& Mason 1997; Dwivedi et al. 1999; Feldman \& Widing 2003; Feldman et al. 2004).

External AR loops rooted in unipolar areas, known as spikes at the edges of ARs (Young \& Mason 1997, 1998) or Mg IX sprays (Sheeley 1996) in earlier studies, were measured to have very high values of FIP bias. More recently, Brooks \& Warren (2011) derived an FIP bias of 3.4 for the upflow regions of AR 10978 over a 5 day period in 2007 December. The FIP bias derived by Brooks \& Warren (2011) of the upflows from the western side of AR 10978 was found to match the value measured in situ a few days later, thus providing observational evidence that upflows become outflows. In general, measurements of the slow SW and solar energetic particles (SEPs) established that lowFIP elements are enriched by a factor of more than 3-4 (Meyer 1985a, 1985b; Gloeckler \& Geiss 1989; Feldman 1992; Reames 

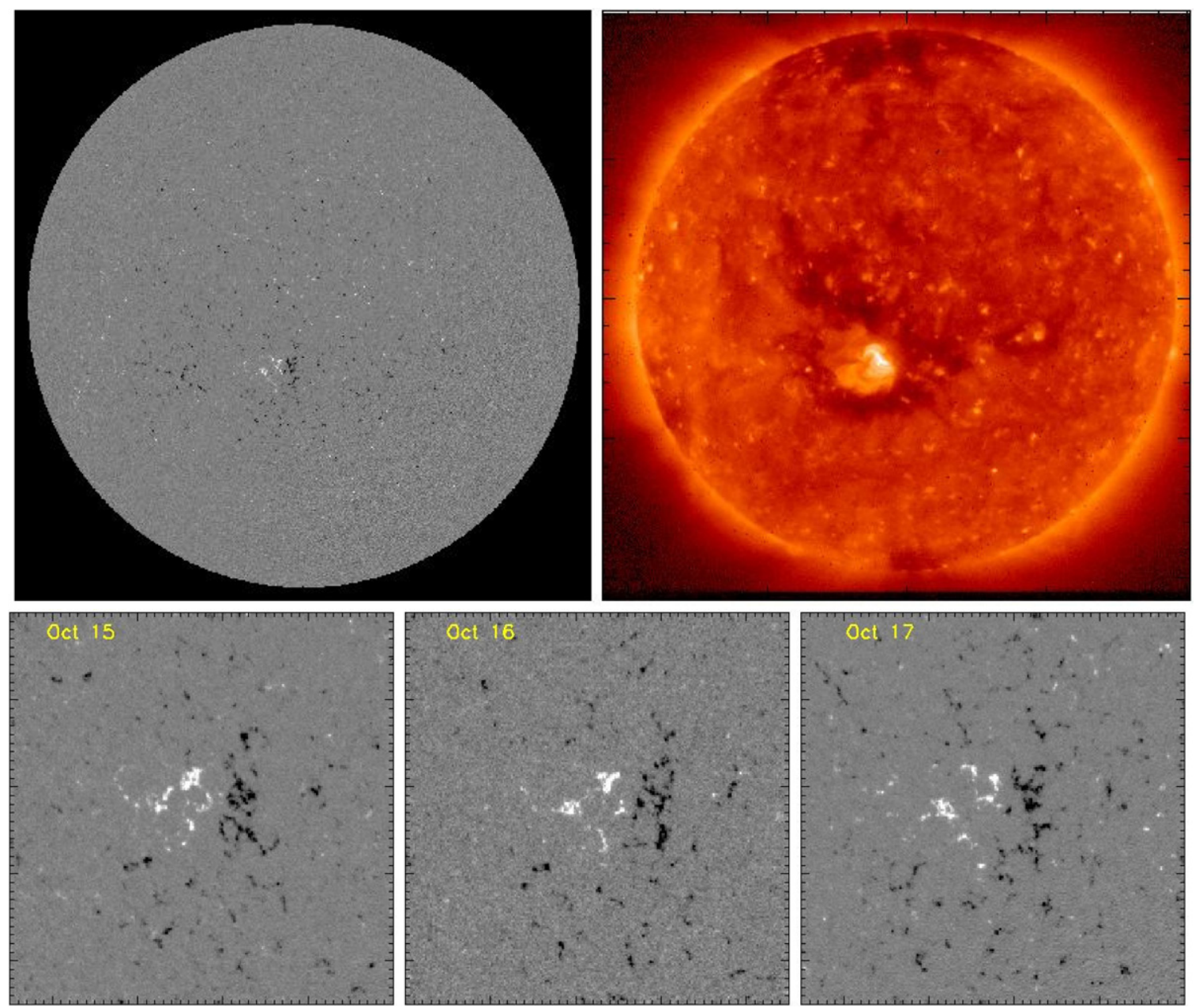

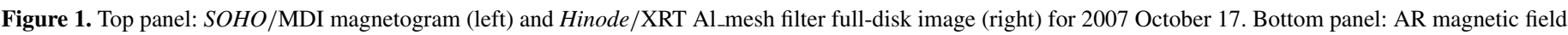
evolution from 2007 October 15 to 17 (saturated at $\pm 250 \mathrm{G}$ ).

(A color version of this figure is available in the online journal.)

1998), comparable to levels observed in ARs and streamers over the activity belt (Raymond et al. 1997; Feldman et al. 1998). See the excellent review paper by Feldman \& Widing (2003) and the references therein for a more complete discussion of elemental abundances and their variations in the solar atmosphere.

Prior to the launch of the EUV Imaging Spectrometer (EIS; Culhane et al. 2007) on board Hinode, individual coronal structures were not easily distinguishable because of lower instrumental spatial resolution and limited fields of view (FOVs; Feldman et al. 2009); consequently, previous elemental abundance studies provided FIP bias levels based on average compositions in coronal QS and $\mathrm{CH}$ regions or small patches of ARs (e.g., Fletcher et al. 2001). In this paper, we present a detailed spatially resolved abundance map of an AR-CH complex that covers an area of $359^{\prime \prime} \times 485^{\prime \prime}$. In the next section, we provide a brief description of the EIS observations of an AR-CH complex from 2007 October 17. In Section 3, we give an account of how the abundance map is derived using the S x $\lambda 264.223$ and Si $x$ $\lambda 258.375$ lines observed by EIS. Our results are presented in Section 4, where we identify the FIP bias levels in the various coronal structures observed in the abundance map and show correlation plots of plasma density, absolute value of magnetic flux density, and nonthermal and Doppler velocities versus FIP bias for the AR in the AR-CH complex. We discuss the im- plications of our results in Section 5 and draw conclusions in Section 6.

\section{OVERVIEW OF AR-CH COMPLEX OBSERVATIONS}

A small "anemone" AR inside a low-latitude $\mathrm{CH}$ was observed close to central meridian on 2007 October 17. Anemone ARs are usually associated with emerging flux within unipolar regions, especially CHs (e.g., Asai et al. 2008; Baker et al. 2009). During emergence, the AR interacts with the ambient coronal field and the AR's magnetic connectivities are reorganized via interchange reconnection. Newly created magnetic loops extend radially from the location of the included AR polarity, thus creating the characteristic anemone configuration. In this event, the AR's included positive polarity reconnects with the surrounding negative field of the $\mathrm{CH}$, forming new compact loops on the AR's eastern side at the interface of the oppositely aligned field. The new loops are evident in the Hinode/X-Ray Telescope (XRT) full-disk image in Figure 1 (top right). The overall magnetic configuration and 3 day on-disk temporal evolution of the AR-CH complex are shown in Solar and Heliospheric Observatory $(\mathrm{SOHO})$ /Michelson Doppler Imager (MDI) full-disk and zoomed magnetograms in Figure 1.

A complete description of this event is given in Baker et al. (2012); however, in this study we focus on a single Hinode/EIS 

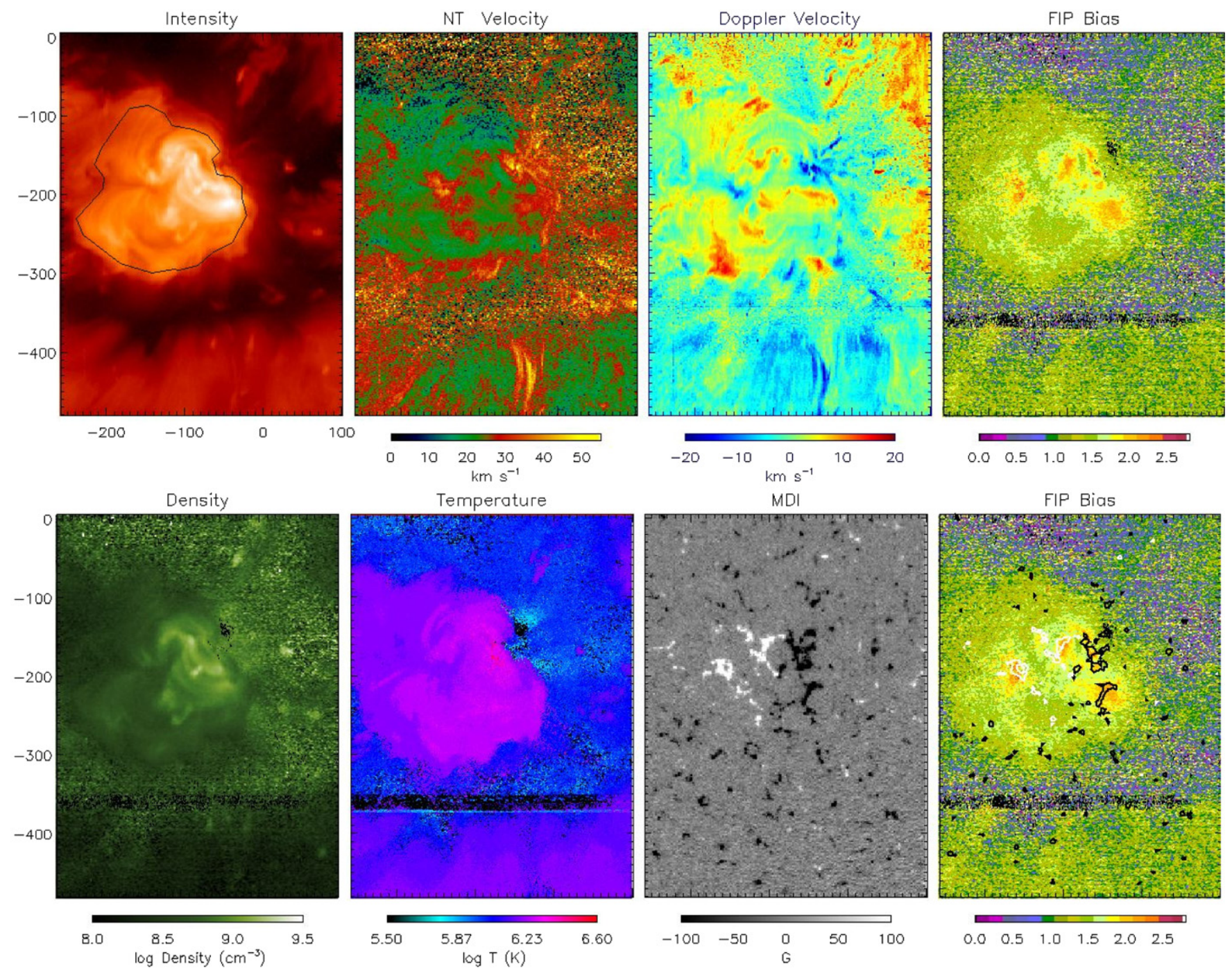

Figure 2. Top panel (left to right): EIS Fe XII intensity, nonthermal velocity, Doppler velocity, and abundance maps for 2007 October 17 at $02: 47$ UT. Bottom panel (left to right): EIS Fe XIII density and temperature maps, MDI magnetogram closest to the EIS raster time (saturation is $\pm 100 \mathrm{G}$ ), and abundance map overlaid with $\pm 100 \mathrm{G}$ MDI contours. $X$ and $Y$ axes are in arcseconds.

(A color version of this figure is available in the online journal.)

raster scan timed at 02:47 UT on October 17. EIS observed the AR-CH complex using the slit scanning mode with the $2^{\prime \prime}$ slit and $2^{\prime \prime}$ step size for 180 pointing positions to build up an FOV of $360^{\prime \prime} \times 512^{\prime \prime}$. Total raster time of $2 \frac{1}{4} \mathrm{hr}$ is composed of $45 \mathrm{~s}$ exposure time at each pointing position. Data reduction was carried out using standard SolarSoft EIS procedures. Raw data were corrected for dark current; hot, warm, and dusty pixels; and cosmic rays. Instrumental effects of slit tilt, CCD detector offset, and orbital variation were corrected. Calibrated spectra were fitted with a single Gaussian function. Reference wavelengths were taken from the average value of a relatively quiescent region along the bottom of the raster. Among the many emission lines simultaneously observed within the EIS spectral bands, we primarily use the Fe XII $\lambda 195.12$ line for intensity, Doppler and nonthermal velocity maps, Fe XIII $\lambda \lambda 202.02$ and 203.83 line pair for the density map, and S x $\lambda 264.223$ and $\mathrm{Si} x \lambda 258.375$ lines and various $\mathrm{Fe}$ ions for constructing the abundance map. EIS Fe XII intensity, nonthermal and Doppler velocity, and S X-Six abundance maps are shown in the top panel of Figure 2. Along the bottom panel of Figure 2, the
Fe XIII density map, temperature map, MDI magnetogram, and abundance map overlaid with MDI $\pm 100 \mathrm{G}$ contours are displayed.

\section{ABUNDANCE MAPS}

To construct the abundance map, we first prepared coaligned intensity images for all the spectral lines we used. This was done by calculating the spatial displacement between short- and long-wavelength CCDs for each wavelength and extracting the common area $\left(359^{\prime \prime} \times 485^{\prime \prime}\right)$. We then fitted Gaussian functions to a series of strong spectral lines from consecutive ionization stages of Fe VIII-XVI. Most of the lines used are unblended and so were fit to single Gaussian functions. In a few cases the line is blended, e.g., Fe XII $\lambda 195.119$ (Del Zanna \& Mason 2005), so multiple Gaussian fits are more appropriate. We also fitted the S x $\lambda 264.223$ and Si $x \lambda 258.375$ lines to be used for the abundance measurement.

The density in each pixel was then measured using the Fe XIII $\lambda 202.044 / \lambda 203.826$ diagnostic ratio, and contribution functions for all the spectral lines were calculated assuming 

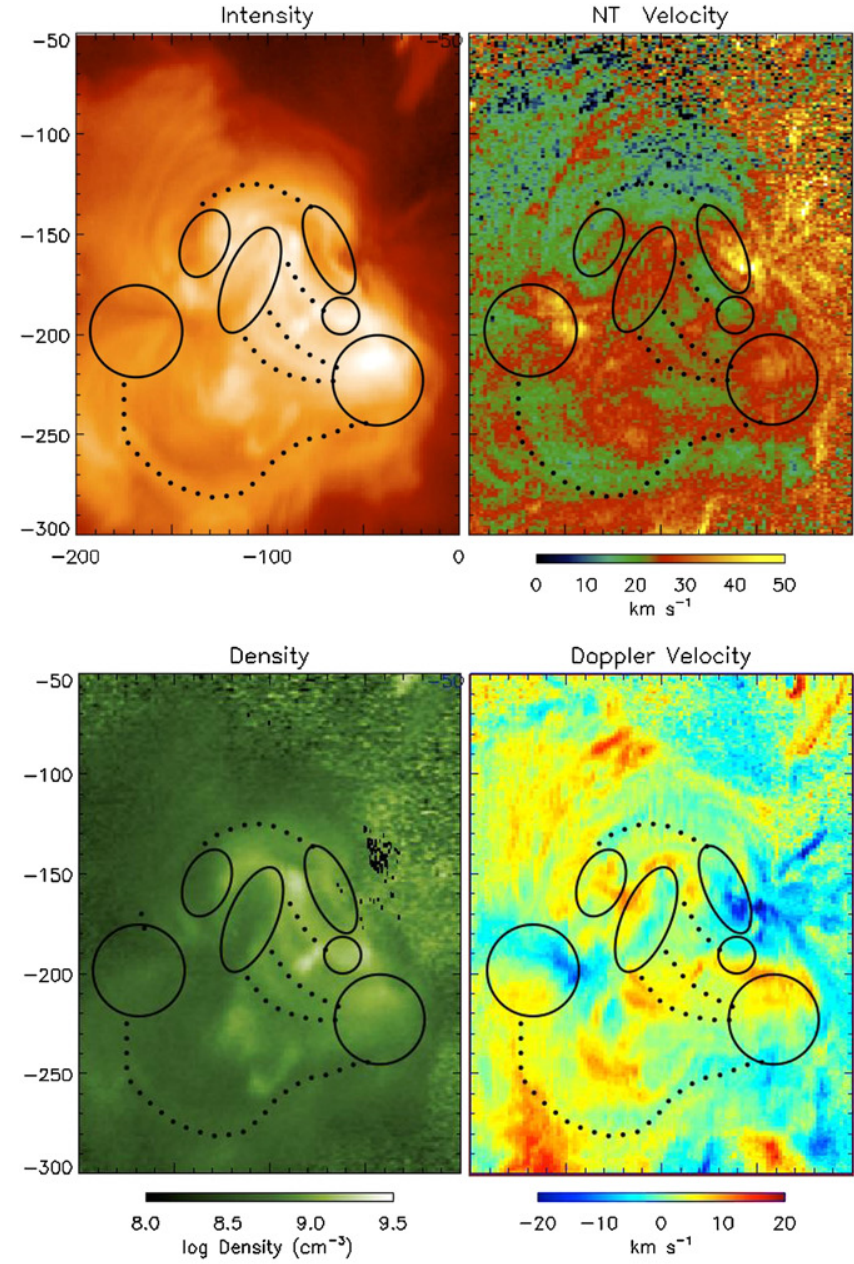

MDI
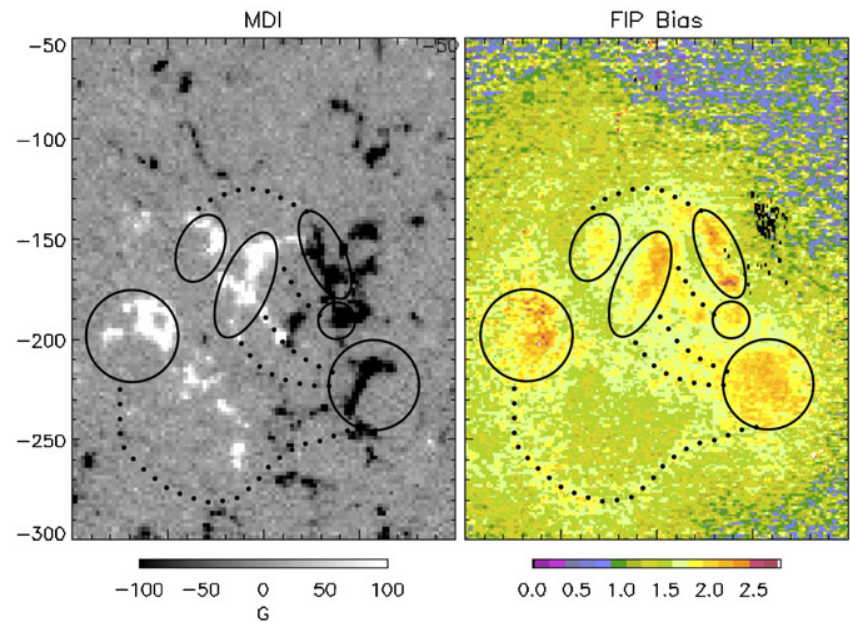

Figure 3. Zoomed AR-CH complex Fe XII intensity and nonthermal velocity maps (top panel), Fe XIII density and Fe XII Doppler velocity maps (middle panel), and MDI magnetogram and abundance map (bottom panel), all showing locations of high FIP bias at loops footpoints (solid black ellipses) and loop traces of enhanced FIP bias (dotted black curves). $X$ and $Y$ axes are in arcseconds.

(A color version and an animation of this figure are available in the online journal.)

this density. We have used the CHIANTI database (Dere et al. 1997; Landi et al. 2012) for calculation of the contribution functions, adopting the photospheric abundances of Grevesse et al. (2007). An emission measure (EM) distribution was then calculated for every pixel using a Markov Chain Monte
Carlo (MCMC) algorithm that calculates alternative solutions by randomly perturbing the observed intensities (Kashyap \& Drake 1998). The EM distributions are convolved with the contribution functions and fitted to the observed intensities to determine the best solution. We compute 100 realizations of the solution for each of the 170,000 pixels in the raster.

The EM is computed using the 10 low-FIP Fe lines only. Since $\mathrm{Si}$ is also a low-FIP element, the derived EM should reproduce the intensity of the Six $\lambda 258.375$ line well. Any mismatch, however, is removed by automatically scaling the EM to reproduce the Six line intensity. Once the best fit for each pixel is found, the FIP bias is calculated as the ratio of the predicted to observed intensity for the S x $\lambda 264.233$ line. Any temperature and density sensitivity of the ratio is accounted for by this method.

\section{RESULTS}

FIP bias of large-scale features within the AR-CH complex can be identified in the spatially resolved abundance map displayed in Figure 2. The surrounding $\mathrm{CH}$ is clearly photospheric in composition with an FIP bias of $\sim 1$, in agreement with previous studies (Feldman et al. 1998; Brooks \& Warren 2011). FIP bias levels within parts of the anemone AR are $>2$. Though the overall FIP bias in this AR is somewhat low compared with previous studies of individual AR features (see Table 1), it is clearly above the CH FIP bias. Quiet Sun FIP bias in the areas surrounding the outer edge of the $\mathrm{CH}$ varies between 1 and 1.5.

Poor signal-to-noise ratio prevents detailed analysis of FIP bias fine structure within the $\mathrm{CH}$; however, there is clear evidence of fine structure within the anemone AR. High FIP bias is concentrated in a few patches located very close to regions of relatively strong magnetic flux density at coronal loop footpoints. This is demonstrated in Figure 2, bottom right panel, where MDI contours of $\pm 100 \mathrm{G}$ are overlaid on the abundance map. FIP bias values are $\sim 2.5-3$ in these regions. In addition, slightly enhanced FIP bias, between 2 and 2.5, appears to trace coronal loops connecting opposite polarity magnetic flux.

Comparison of the maps in Figure 2 suggests morphological groupings of FIP bias structures. This is examined more closely in Figure 3. High FIP bias patches are spatially coincident with internal AR loop footpoints in the Fe XII intensity and nonthermal maps. The dotted lines of slightly enhanced FIP bias appear to trace very well loops connecting bipoles composing the AR (see the animation abund_intensity.mp4 included as an electronic supplement to this article). These magnetic connections do not represent newly reconnected loops between emerging $\mathrm{AR}$ and surrounding $\mathrm{CH}$ loops but original AR connectivities. They can be regarded as old loops.

The abundance map is further zoomed in Figure 4 to highlight a channel of low FIP bias in between the westernmost ellipses to the AR's N/NW in the lower right panel of Figure 3. This region of lower FIP bias is cospatial with the main magnetic polarity inversion line (PIL) within the AR, along which the bright coronal loops become increasing sheared and sigmoidal in soft X-rays by 18:00 UT on the 17th and eventually erupt at 07:30 UT on the 18th (Baker et al. 2012).

Possible links between FIP bias in the anemone AR and plasma parameters are better illustrated with correlation plots constructed using the maps in Figure 2. Data from pixels within a contour fitted around the AR were extracted for intensity, nonthermal and Doppler velocities, density, temperature, and absolute magnetic flux density. The contour is overplotted on the intensity map of the AR- $\mathrm{CH}$ complex in Figure 2. Pixels with 
Table 1

FIP Bias in Solar Structures

\begin{tabular}{|c|c|c|}
\hline Structure & FIP Bias & Reference \\
\hline Coronal holes & $\sim 1, \sim 2,1.2$ & $1,2,3,4$ \\
\hline Plumes & 9.4 & 5 \\
\hline \multirow[t]{2}{*}{ Quiet Sun } & $1.5-2.0$ & 1 \\
\hline & $\sim 2,2.3,3.5$ & $1,6,7$ \\
\hline Streamers & $\sim 3, \sim 4$ & 8,2 \\
\hline Surges & Photospheric & 9 \\
\hline \multicolumn{3}{|l|}{ Prominences/Filaments } \\
\hline Quiescent & $1.1-2.4$ & 10 \\
\hline Eruptive & Photospheric & 9 \\
\hline \multicolumn{3}{|l|}{ Flares } \\
\hline Impulsive & $\sim 1$ & 11,12 \\
\hline B7.3-X1.5 & $2.4-2.6$ & 13 \\
\hline SEPs & $\sim 4-6$ & $14,15,16,17$ \\
\hline \multicolumn{3}{|l|}{ Active regions } \\
\hline Emerging & Photospheric & $12,18,19,20$ \\
\hline Established (a few days) & $4.8-5.9$ & 21 \\
\hline Old ( $>7$ days $)$ & $8-16$ & $17,21-25$ \\
\hline TR brightenings-BPs ${ }^{\mathrm{a}}$ & Photospheric & 26 \\
\hline TR brightenings-QSLs ${ }^{\mathrm{b}}$ & $2-4$ & 26 \\
\hline Core loop & Photospheric & 22 \\
\hline Spikes at edges/Mg IX sprays & 4-11 & $19,22,27$ \\
\hline Upflows & 3.4 & 4 \\
\hline High-speed blue-wing upflows & $3-5$ & 28 \\
\hline \multicolumn{3}{|l|}{ Solar wind } \\
\hline Fast & Photospheric & 29 \\
\hline Slow & Coronal & 29 \\
\hline \multicolumn{3}{|l|}{ Open $B$-field on disk } \\
\hline$>14 \%$ & $1.7-2.5$ & 30 \\
\hline$<7 \%$ & $2.8-4.2$ & 30 \\
\hline
\end{tabular}

Notes.

${ }^{a}$ BP: bald patch separatrices.

b QSLs: quasi-separatrix layers.

References: (1) Feldman \& Widing 1993; (2) Feldman et al. 1998; (3) Doschek et al. 1998; (4) Brooks \& Warren 2011; (5) Widing \& Feldman 1992; (6) Warren 1999; (7) Landi et al. 2006; (8) Raymond et al. 1997; (9) Widing et al. 1986; (10) Spicer et al. 1998; (11) McKenzie \& Feldman 1992; (12) Widing 1997; (13) Sylwester et al. 2013; (14) Meyer 1985a; (15) Meyer 1985b; (16) Reames 1998; (17) Feldman 1992; (18) Sheeley 1995; (19) Sheeley 1996; (20) Widing \& Feldman 2001; (21) Widing \& Feldman 1995; (22) Young \& Mason 1997; (23) Dwivedi et al. 1999; (24) Feldman \& Widing 2003; (25) Feldman et al. 2004; (26) Fletcher et al. 2001; (27) Young \& Mason 1998; (28) Brooks \& Warren 2012; (29) Gloeckler \& Geiss 1989; (30) Wang et al. 2009.

a Doppler velocity in between $\pm 5 \mathrm{~km} \mathrm{~s}^{-1}$ were removed so that only relatively strong flows in excess of the EIS error are included. Furthermore, a reduced chi-squared filter based on the EM calculation was applied to the data. The filter removes pixels that deviate too strongly from the fitted EM $\left(\bar{\chi}^{2}<2\right)$ without being too stringent in the case of FIP bias values.

Selected plasma parameter data are plotted versus FIP bias in Figure 5. The data are fitted with a linear relationship, and correlation coefficients are given in each correlation plot. Overall, there is a moderate positive correlation of three plasma parameters with FIP bias in the AR: nonthermal velocity, (correlation coefficient of 0.36$)$, density $(0.44$; intensity is similarly correlated with FIP bias), and absolute value of magnetic flux density (0.37). Temperature is weakly correlated with FIP bias (correlation coefficient of 0.20 ; not shown). Doppler velocity, whether downflow or upflow, does not appear to be correlated with FIP bias. Note that the method utilized in constructing the abundance map is designed to remove any effect of temperature and density on FIP bias measurement;

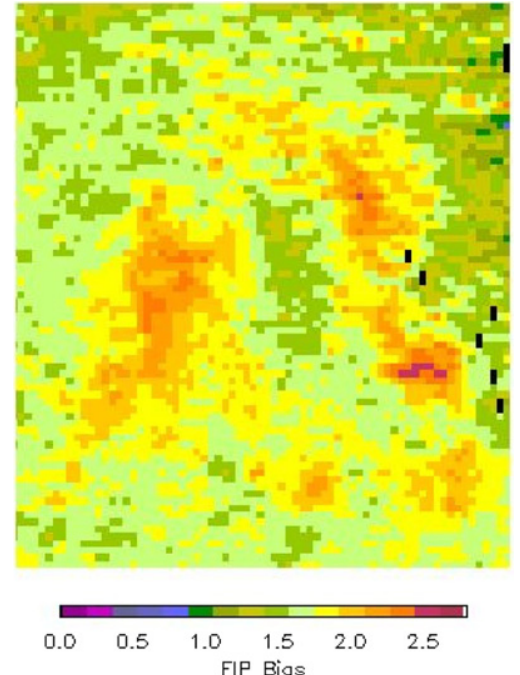

Figure 4. Zoomed abundance map of the inverse S-shaped sigmoid channel of low FIP bias along the main PIL hosting a filament within the AR where a sigmoid/flux rope forms and eventually erupts (Baker et al. 2012).

(A color version of this figure is available in the online journal.)

therefore, the moderate positive correlation of the measured FIP bias with density and the weak correlation with temperature are expected to be real and due to the underlying physics in the creation of FIP bias.

\section{DISCUSSION}

Table 1 shows typical FIP bias values for established ARs to be greater than 4, but for this event, the FIP bias enhancement is $2-3$. We suggest that it is likely that the overall relatively low FIP bias levels measured within the AR are attributable to the age and magnetic configuration of the AR-CH complex. In general, large variation in AR FIP bias is related to the variation in the average age of ARs (Widing \& Feldman 2001; McKenzie $\&$ Feldman 1992). New flux emergence is characterized by photospheric composition (Sheeley 1995, 1996; Widing 1997; Widing \& Feldman 2001). Thereafter, the FIP bias of evolving ARs progresses at approximately a constant rate toward coronal levels within days of emergence (Widing \& Feldman 2001). For the studied AR, the low level of FIP bias may be due to the fact that the anemone AR is dominated by recently formed loops instead of the older, more extended, lower density loop structures of mature ARs (Young \& Mason 1997). The age of the AR should not be much more than $\approx 10$ days as its low flux content, $3 \times 10^{21} \mathrm{Mx}$, puts it in the small AR category for which the average lifetime is measured in days (Schrijver \& Zwaan 2008).

The surrounding $\mathrm{CH}$ may also contribute to the low FIP bias levels in the dominantly negative monopolar field. $\mathrm{CH}$ plasma undergoes very little if any modification upon emerging from the photosphere; therefore, its composition remains unchanged at levels close to 1 , as was confirmed by this study. The fact that the AR is fully surrounded by $\mathrm{CH}$ plasma of photospheric composition means that low FIP bias plasma of the $\mathrm{CH}$ can readily mix with the high FIP bias of the AR plasma after interchange reconnection has occurred (forming part of the anemone AR loops). Since anemone ARs form only in the unipolar field of $\mathrm{CHs}$, it is plausible that they would have lower FIP bias levels compared with ARs surrounded by mixed polarity QS plasma of higher FIP bias, assuming that other 

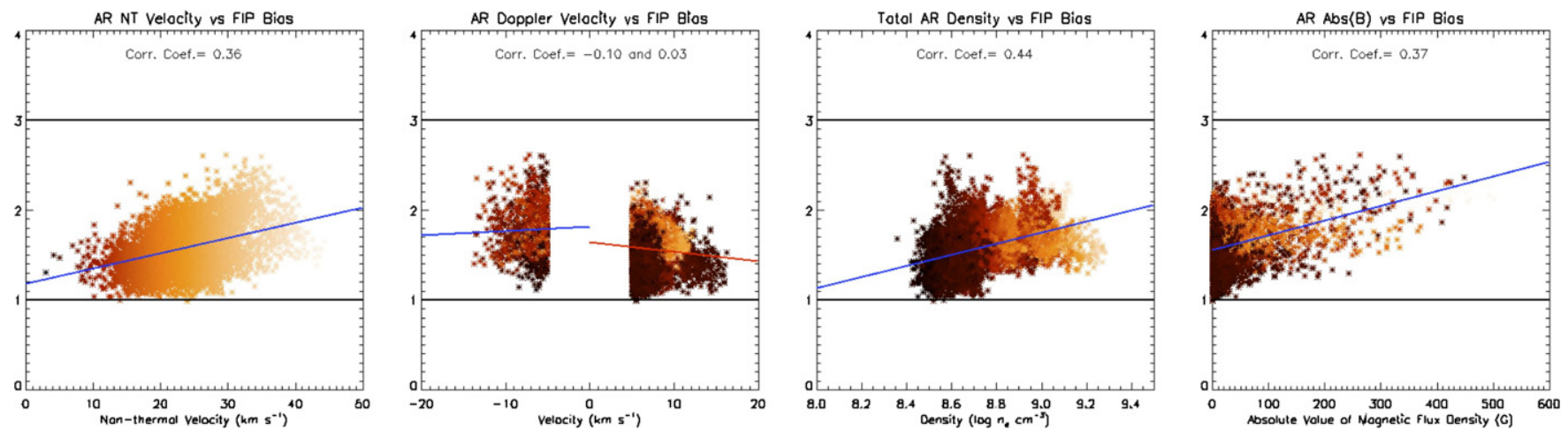

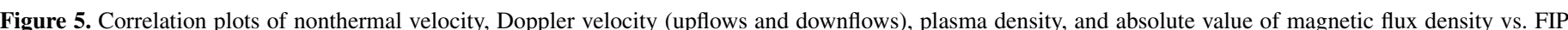

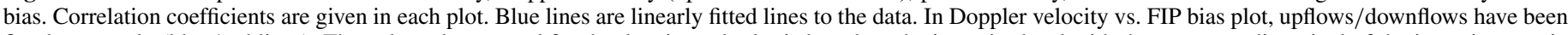

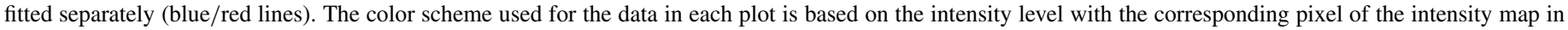
Figure 2.

(A color version of this figure is available in the online journal.)

factors such as age are similar between the ARs. This is consistent with the results of Wang et al. (2009), who found that over solar cycle 23 , the average enrichment factor compared to photospheric values for a group of low FIP elements, including $\mathrm{Si}$, was only 1.7-2.5 when the fraction of open flux on the visible side of the solar disk exceeded 14\%; however, the enrichment factor increased to 2.8-4.2 when the open flux fraction was less than $7 \%$.

The internal structure of the AR consists of higher FIP bias loops and a low FIP bias channel around the main PIL of the AR. This low FIP bias region could a priori be due to local flux emergence all along the PIL; however, such emergence will only consist of small, local bipoles that would be covered over by a set of coronal loops. The FIP bias in the overlying loops would be expected to be similar to the higher FIP bias levels found in other parts of the AR. Moreover, emergence is not observed along the PIL (Baker et al. 2012); therefore, the emergence hypothesis cannot explain the observed low FIP bias. However, significant flux cancellation along the main AR PIL has been ongoing for several days prior to the time of EIS observation on the $17 \mathrm{th}$. The flux cancellation creates a sigmoid as seen in soft X-rays and is observed at the photosphere as the disappearance of small magnetic bipoles at the PIL occurs (Baker et al. 2012). This implies that reconnection is occurring in the lower atmosphere because very small loops are required for submergence along the PIL (van Ballegooijen \& Martens 1989). This scenario has been used to show that sigmoid formation can indicate the buildup of a flux rope in Green et al. (2011). Green \& Kliem (2009) have shown that the bottom of the flux rope in a sigmoidal region can be located low down in the solar atmosphere as expected from its formation via flux cancellation. This means that field lines running under the flux rope will have a bald patch (BP) topology, where field lines are tangent to the photosphere. Photospheric plasma is able to get access to BP field lines as a result of their creation via reconnection in the lower atmosphere.

Converging and/or shearing motions in the photosphere can then induce the formation of a current sheet all along the separatrix attached to the BP, and typically a current sheet is also formed above the BP (Low \& Wolfson 1988). Then, magnetic reconnection is theoretically expected, as was verified in MHD simulations (e.g., Aulanier et al. 2010). Reconnection at the BP was found to be part of the flux rope buildup process before the flux rope becomes unstable and a coronal mass ejection $(\mathrm{CME})$ is launched. Magnetic reconnection at the BP has the particularity to occur in the cold part of the solar atmosphere and then to input photospheric plasma in upwardly curved field lines, hence lifting up and heating photospheric material in the newly formed longer coronal loops. Such plasma is expected to have photospheric abundances. Indeed, in EUV brightenings, plasma along BP field lines has been shown to have photospheric FIP bias in contrast to quasi-separatrix layer (QSL) field lines (Fletcher et al. 2001). The presence of a low FIP bias sigmoid channel in the anemone AR may indicate that reconnection low in the atmosphere leads to mixing of photospheric composition material with coronal plasma with higher FIP. The photospheric FIP values previously determined from Skylab observations for an eruptive filament (see Table 1), together with the typical presence of BPs below filaments (e.g., Aulanier et al. 1998), are consistent with our results and supportive of a BP topology within the sigmoid channel.

Though it is possible that uncertainties in the method employed to determine FIP bias may be contributing to the low levels measured in the AR, the impact is likely to be minimal when weighed against real factors such as age and magnetic topology of the AR-CH complex. We selected well-tested lines, and the atomic data for these lines are expected to be accurate to derive the FIP bias. Also, EIS has the best temperature and density diagnostics of any coronal instrument to date.

A further convincing test of the FIP bias results is the correspondences found with the observed coronal structures. First, the $\mathrm{CH}$ was determined to have photospheric composition. Second, the largest enhanced FIP bias patches are located at the base of coronal loops. Third, coronal loop connectivities are clearly traced in the abundance map. Finally, the low FIP bias channel around the main AR PIL is consistent with the presence of a sigmoid with a BP topology. All of these correspondences between FIP bias and coronal structures show that the FIP bias uncertainties are well below the observed range of FIP bias variations.

One caveat is that $\mathrm{Si}$ and $\mathrm{S}$ are close to the usually defined boundary between high and low FIP elements. Although the observed variations clearly show that the $\mathrm{Si} / \mathrm{S}$ ratio is sensitive to FIP bias, some models indicate that $\mathrm{Si}$ fractionates relatively less than other low FIP elements and S fractionates relatively more than other high FIP elements. This suggests that the actual level of FIP bias could be underestimated (Laming 2012). The observational picture is less clear, however. Evidence suggests that $\mathrm{S}$, for example, behaves like a high-FIP element in ARs 
(Lanzafame et al. 2002), but over-fractionates in quiet regions (Brooks et al. 2009). An anemone AR may represent some intermediate state between the two. Note that our method of scaling the Fe EM to that of $\mathrm{Si}$ allows a calibration of any under-fractionation of Si. In Brooks \& Warren (2011) we found that this scaling was less than $20 \%$, indicating a similar behavior for $\mathrm{Fe}$ and $\mathrm{Si}$, and that the FIP bias values are accurate in many cases.

\section{CONCLUSIONS}

In this paper we analyze an anemone $\mathrm{AR}$ inside an on-disk $\mathrm{CH}$ using observations from Hinode/EIS, XRT, and SOHO/MDI from 2007 October 17. We constructed large-scale $359^{\prime \prime} \times$ $485^{\prime \prime}$ intensity, nonthermal and Doppler velocities, density, temperature, and abundance maps of the AR-CH complex. FIP bias in the surrounding $\mathrm{CH}$ is $\sim 1$, consistent with previous composition studies of $\mathrm{CHs}$, and FIP bias of the anemone AR is $2-3$, which is lower than those values obtained from AR composition studies listed in Table 1.

No consensus exists as to a single mechanism of cause for the FIP effect; however, it is generally accepted that fractionation takes place in the chromosphere where low-FIP elements are mainly ionized and high-FIP elements are at least partially neutral. Widing \& Feldman (2001) identified the footpoints and legs of loop-like structures as the location where fractionation and uplift occur. We find strong evidence in support of Widing \& Feldman (2001) in the anemone AR, where high FIP bias is distinctly concentrated at the AR's loop footpoints (see ellipses in Figure 3). In a young AR, there is insufficient time for highFIP plasma to fill the coronal loops, so the concentration of high FIP bias at the footpoints suggests that FIP bias enhancement begins at the AR's footpoints, in close proximity to where fractionation occurs.

We detect the start of high FIP bias plasma mixing in some of the coronal loops in the abundance map in Figure 3. Pathways of slightly enhanced FIP bias are traced along loops connecting opposite polarities of bipolar magnetic concentrations within the AR, indicating that the loops are partly filled with the high FIP bias plasma. The degree of mixing of plasma along the loops is expected to be limited in this case due to the relatively weak heating generated by the weak mean magnetic field of the anemone $\mathrm{AR}$, which was measured to be $\approx 80 \mathrm{G}$ when it crossed the solar central meridian (Baker et al. 2012). Furthermore, when the anemone $\mathrm{AR}$ and nearby $\mathrm{CH}$ fields reconnect, new loops form that are similar in size to the AR loops, so enhanced FIP bias transferred to the reconnected loops is partly mixed with previously open-field plasma of lower FIP bias. The enhanced FIP bias is not entirely diluted because the loop size is similar to that of the pre-reconnection loops.

We also found a moderate positive correlation of high FIP bias with nonthermal velocity and the absolute value of magnetic flux density. Our observations favor FIP effect models that are located in the vicinity of AR footpoints from where the fractionated plasma is then transported throughout the loops by diffusion. One such model is the Laming FIP effect model (Laming 2004, 2009, 2012), which invokes the ponderomotive force arising from Alfvén waves of coronal origin reflecting from the chromosphere at loop footpoints and the induced generation of slow-mode waves to explain FIP fractionation. If we interpret the strong nonthermal velocity in the AR's footpoints to be a slow-mode wave along the magnetic field, then the slow-mode amplitudes of the Laming (2012) model are comparable to observed nonthermal velocities (see Laming 2012, Figure 8).
Finally, the low FIP bias observed within the inverse S-shaped sigmoid channel just above the AR's PIL is atypical of the global pattern of the AR FIP bias. This low FIP bias cannot be due to emergence since it is not observed. Rather, we propose that low FIP bias in the sigmoidal channel could be the underbelly of a flux rope formed by flux cancellation along the PIL, which is highly suggestive of a BP topology. In such a configuration, reconnection takes place at the photospheric level, lifting up photospheric plasma in the magnetic dips, implying a low FIP bias when mixed with the coronal plasma of the reconnecting loops. To date, determining the particular magnetic topology of a sigmoid/flux rope configuration has proved to be problematic because both coronal loops and photospheric magnetic field are nearly aligned with the PIL so that it is difficult to determine if the magnetic configuration is normal or inverse (with curved down or up field lines, respectively). FIP bias provides key evidence to clearly distinguish between BP and QSL topology, independent of direct observations of the magnetic field. This has far-reaching implications for predicting CMEs as BP reconnection is expected to be present well before a CME, as shown in MHD numerical simulations. Identifying a BP topology where reconnection is occurring allows for the identification of the early building up of a flux rope that later will become unstable and will create a CME if the overlying magnetic field is not too strong (otherwise it is a failed eruption). Then, we conclude that the abundance maps have the potential to identify the early formation of flux ropes that are potential sites of CMEs.

Hinode is a Japanese mission developed and launched by ISAS/JAXA, collaborating with NAOJ as a domestic partner, NASA and STFC (UK) as international partners. Scientific operation of Hinode is by the Hinode science team organized at ISAS/JAXA. This team mainly consists of scientists from institutes in the partner countries. Support for the post-launch operation is provided by JAXA and NAOJ (Japan), STFC (UK), NASA, ESA, and NSC (Norway). L.v.D.-G. and K.S. acknowledge the European Community FP7/2007-2013 programme through the eHEROES Network (EU FP7 Space Science Project no. 284461). K.S. also acknowledges support from the SWIFF Network (EU FP7 Space Science Project no. 263340). L.v.D.-G. acknowledges the Hungarian government for grant OTKA K081421. The work of D.H.B. was performed under contract with the Naval Research Laboratory and was funded by the NASA Hinode program. J.C. thanks UCL and the Max Planck Institute for an Impact Studentship award. D.B.'s work was supported by STFC. We thank the referee for his/her constructive and inspiring comments, which have improved the article.

\section{REFERENCES}

Asai, A., Shibata, K., Hara, H., \& Nitta, N. V. 2008, ApJ, 673, 1188

Aulanier, G., Démoulin, P., van Driel-Gesztelyi, L., Mein, P., \& Deforest, C. 1998, A\&A, 335, 309

Aulanier, G., Török, T., Démoulin, P., \& DeLuca, E. E. 2010, ApJ, 708, 314

Baker, D., Rouillard, A. P., van Driel-Gesztelyi, L., et al. 2009, AnGeo, 27, 3883

Baker, D., van Driel-Gesztelyi, L., \& Green, L. M. 2012, SoPh, 276, 219

Brooks, D. H., \& Warren, H. P. 2011, ApJL, 727, L13

Brooks, D. H., \& Warren, H. P. 2012, ApJL, 760, L5

Brooks, D. H., Warren, H. P., Williams, D. R., \& Watanabe, T. 2009, ApJ, 705,1522

Culhane, J. L., Harra, L. K., James, A. M., et al. 2007, SoPh, 243, 19

Del Zanna, G., \& Mason, H. E. 2005, A\&A, 433, 731

Dere, K. P., Landi, E., Mason, H. E., Monsignori Fossi, B. C., \& Young, P. R. 1997, A\&AS, 125, 149

Doschek, G. A., Laming, J. M., Feldman, U., et al. 1998, ApJ, 504, 573 
Dwivedi, B. N., Curdt, W., \& Wilhelm, K. 1999, ApJ, 517, 516

Feldman, U. 1992, PhyS, 46, 202

Feldman, U., Dammasch, I., Landi, E., \& Doschek, G. A. 2004, ApJ, 609, 439

Feldman, U., Schühle, U., Widing, K. G., \& Laming, J. M. 1998, ApJ, 505, 999

Feldman, U., Warren, H. P., Brown, C. M., \& Doschek, G. A. 2009, ApJ, 695, 36

Feldman, U., \& Widing, K. G. 1993, ApJ, 414, 381

Feldman, U., \& Widing, K. G. 2002, PhPl, 9, 629

Feldman, U., \& Widing, K. G. 2003, SSRv, 107, 665

Fletcher, L., López Fuentes, M. C., Mandrini, C. H., et al. 2001, SoPh, 203, 255

Gloeckler, G., \& Geiss, J. 1989, in AIP Conf. Proc. 183, Cosmic Abundances of Matter, ed. C. J. Waddington (Melville, NY: AIP), 49

Green, L. M., \& Kliem, B. 2009, ApJL, 700, L83

Green, L. M., Kliem, B., \& Wallace, A. J. 2011, A\&A, 526, A2

Grevesse, N., Asplund, M., \& Sauval, A. J. 2007, SSRv, 130, 105

Kashyap, V., \& Drake, J. J. 1998, ApJ, 503, 450

Laming, J. M. 2004, ApJ, 614, 1063

Laming, J. M. 2009, ApJ, 695, 954

Laming, J. M. 2012, ApJ, 744, 115

Landi, E., Del Zanna, G., Young, P. R., Dere, K. P., \& Mason, H. E. 2012, ApJ, 744, 99

Landi, E., Feldman, U., \& Doschek, G. A. 2006, ApJ, 643, 1258

Lanzafame, A. C., Brooks, D. H., Lang, J., et al. 2002, A\&A, 384, 242

Low, B. C., \& Wolfson, R. 1988, ApJ, 324, 574
McKenzie, D. L., \& Feldman, U. 1992, ApJ, 389, 764

Meyer, J.-P. 1985a, ApJS, 57, 173

Meyer, J.-P. 1985b, ApJS, 57, 151

Meyer, J.-P. 1991, AdSpR, 11, 269

Raymond, J. C., Kohl, J. L., Noci, G., et al. 1997, SoPh, 175, 645

Reames, D. V. 1998, SSRv, 85, 327

Schrijver, C. J., \& Zwaan, C. 2008, Solar and Stellar Magnetic Activity (Cambridge: Cambridge Univ. Press)

Sheeley, N. R., Jr. 1995, ApJ, 440, 884

Sheeley, N. R., Jr. 1996, ApJ, 469, 423

Spicer, D. S., Feldman, U., Widing, K. G., \& Rilee, M. 1998, ApJ, 494, 450

Sylwester, B., Phillips, K. J. H., Sylwester, J., \& Kȩpa, A. 2013, SoPh, 283, 453

van Ballegooijen, A. A., \& Martens, P. C. H. 1989, ApJ, 343, 971

van Driel-Gesztelyi, L., Culhane, J. L., Baker, D., et al. 2012, SoPh, 281, 237

Wang, X., Klecker, B., \& Wurz, P. 2009, A\&A, 505, 1237

Warren, H. P. 1999, SoPh, 190, 363

Widing, K. G. 1997, ApJ, 480, 400

Widing, K. G., \& Feldman, U. 1992, ApJ, 392, 715

Widing, K. G., \& Feldman, U. 1995, ApJ, 442, 446

Widing, K. G., \& Feldman, U. 2001, ApJ, 555, 426

Widing, K. G., Feldman, U., \& Bhatia, A. K. 1986, ApJ, 308, 982

Woo, R., Habbal, S. R., \& Feldman, U. 2004, ApJ, 612, 1171

Young, P. R., \& Mason, H. E. 1997, SoPh, 175, 523

Young, P. R., \& Mason, H. E. 1998, SSRv, 85, 315 